\title{
A Novel Prior Noise Correction - Adaptive Extended Kalman Filtering Method for the Full Parameter and State-of-energy co- estimation of the Lithium-ion Batteries
}

\author{
Lili Xia, Shunli Wang ${ }^{*}$, Chunmei Yu, Cong Jiang, Yongcun Fan, Wen Cao \\ School of Information Engineering, Southwest University of Science and Technology, Mianyang \\ 621010, China; \\ *E-mail: $\underline{497420789 @ q q . c o m}$
}

doi: $10.20964 / 2021.07 .30$

Received: 28 February 2021 / Accepted: 9 April 2021 / Published: 31 May 2021

\begin{abstract}
In the battery management system, the state-of-energy is an important state to represent the remaining energy of the battery. The equivalent circuit model is the key to predicate this state of the lithium-ion battery. Therefore, the modeling and parameter identification of the battery model is crucial. This paper proposes a full parameter identification algorithm based on the forgetting factor recursive extended leastsquare algorithm, which is leveraged to calculate parameters including the open-circuit voltage of the equivalent circuit model. Besides, the prior noise correction adaptive extended Kalman filtering algorithm is derived to predict the state-of-energy with the proposed full parameters identification algorithm. The prior noise correction is an efficient method to reduce the estimation error of the extended Kalman filtering algorithm, which predicts the noise at the next moment by current noise. Comparing with the extended Kalman filtering algorithm, the noise of prior noise correction adaptive extended Kalman filtering algorithm can be corrected efficiently. In this way, the maximum error of the forgetting factor recursive extended least-square algorithm to estimate open-circuit-voltage is $0.41 \%$ under different complex working conditions comparing with actual values. The modeling accuracy by full parameters identification is higher than $99.31 \%$. For verification of state-of-energy, two different complexes working conditions are conducted to calculated state-of-energy, the error of state-of-energy estimation is less than $1.49 \%$. The results demonstrate that the proposed algorithm can perfect the state estimation.
\end{abstract}

Keywords: lithium-ion battery; state-of-energy; adaptive extended Kalman filtering; battery management system; parameter identification;

FULL TEXT 
(C) 2021 The Authors. Published by ESG (www.electrochemsci.org). This article is an open access article distributed under the terms and conditions of the Creative Commons Attribution license (http://creativecommons.org/licenses/by/4.0/). 